\title{
Induction of type-IIA secretory phospholipase A2 in animal models of acute lung injury
}

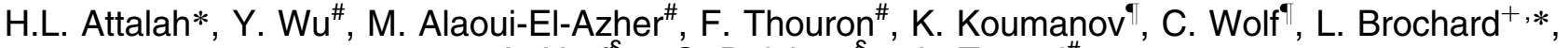 \\ A. Harf ${ }^{\S, *}$, C. Delclaux ${ }^{\S, *}$, L. Touqui ${ }^{\#}$
}

Induction of type-IIA secretory phospholipase A2 in animal models of acute lung injury. H.L. Attalah, Y. Wu, M. Alaoui-El-Azher, F. Thouron, K. Koumanov, C. Wolf, L. Brochard, A. Harf, C. Delclaux, L. Touqui. C) ERS Journals Ltd 2003.

ABSTRACT: The aim of this study was to evaluate the presence of type-II secretory phospholipase A2 (sPLA2-IIA) in alveolar space and its possible role in the destruction of surfactant in three rat models of acute lung injury.

Alveolar instillation of either lipopolysaccaride or live Pseudomonas aeruginosa resulted in a significant increase in lung oedema and in a decrease in static compliance of the respiratory system together with alveolar-neutrophil influx as compared with healthy control rats.

The upregulation of messenger ribonucleic acid and SPLA2-IIA by the lung was evident. This was associated with surfactant degradation and a decrease in large:small ratio of surfactant aggregates in bacteria-instilled rats. A negative correlation between compliance and SPLA2-IIA activity in bronchoalveolar lavage fluid was shown. By contrast, during alpha naphthylthiourea-induced injury, neither alveolar-neutrophil influx nor increase in SPLA2-IIA activity was observed.

Additional experiments in rats treated with a specific inhibitor of type-II secretory phospholipase $\mathrm{A} 2$ activity (3 acetamine-1-benzyl-2 ethylindolyl-5 oxy; propane phosphonic acid (LY311727)) demonstrated no improvement in physiological parameters despite a biochemical effect, suggesting that its activity is only one of the multiple factors involved in the pathophysiology of lung injury.

Eur Respir J 2003; 21: 1040-1045.
*Unité Institut National de la Santé et de la recherche médicale (INSERM) U.492, Faculté de Médecine de Créteil-Université Paris XII, ${ }^{\#}$ Unité de Défense Innée et Inflammation, Institut Pasteur, Paris, Laboratoire de Biochimie, Hôpital St Antoine, AP-HP, ${ }^{+}$Service de Réanimation Médicale and ${ }^{\S}$ Service de Physiologie-Explorations Fonctionnelles, Hôpital Henri Mondor, AP-HP, France.

Correspondence: C. Delclaux, Unité INSERM U492, Faculté de Médecine de Créteil, 8, rue du Général Sarrail, 94000 Créteil, France.

Fax: 33148981777

E-mail: delclaux@im3.inserm.fr

Keywords: Alpha naphthylthiourea compliance

endotoxin

Pseudomonas aeruginosa

Received: October 112002

Accepted after revision: February 42003
Surfactant degradation has been shown to be one of the key features of acute respiratory distress syndrome (ARDS) leading to a decrease in both lung compliance and oxygenation $[1,2]$. Consequently, several clinical trials have evaluated the effect of exogenous surfactant administration. However, the relative inefficacy of this approach has been attributed to insufficient alveolar delivery of exogenous surfactant and/or to its inhibition/degradation by alveolar enzymatic activities [2]. Along this line, type-IIA secretory phospholipase A2 (sPLA2-IIA), an enzyme produced by alveolar inflammatory cells during acute lung injury (ALI), has previously been shown to participate in surfactant degradation [3-5]. SPLA2IIA belongs to a superfamily of enzymes that hydrolyses the $s n-2$ fatty acyl ester bond of phosphoglycerides, generating lysophospholipids and free fatty acids [6]. These enzymes play a major role in the induction of inflammatory reactions via the synthesis of lipid mediators, such as platelet activating factor, leukotrienes and prostaglandins [5, 6]. A number of studies suggest that sPLA2-IIA is involved in the pathogenesis of inflammatory diseases including ARDS. Indeed, a clinical trial demonstrated an increased sPLA2-IIA activity in bronchoalveolar lavage (BAL) fluid of patients with ARDS whose levels were positively correlated with disease severity [5].

The assessment of the role of sPLA2-IIA activity in terms of both surfactant and compliance impairment is of major value, since its inhibition could constitute a therapeutic approach. Therefore, the aims of this study were to assess whether correlation could be established between indexes of injury (lung oedema, arterial oxygenation, lung mechanics) and surfactant degradation in experimental models of ALI. Moreover, the evaluation of three different rat models of ALI allowed the assessment of the respective roles of lung oedema and alveolar inflammation in lung compliance impairment.

\section{Material and methods}

\section{Animals}

Adult male Sprague-Dawley rats (IFFA CREDO Laboratories, L'arbresle, France) weighing 225-250 g were used in all experiments. The rats were housed in air-filtered, temperature-controlled units and were allowed free access to food and water.

\section{Experimental procedures}

Three models of rat ALI, which have been described previously [7-10], were evaluated. 1) The first model consisted of live Pseudomonas aeruginosa in alveolar instillation [7-9]. In these experiments, a low bacterial inoculum was used, leading to no mortality. However, alveolar-neutrophil influx was evident together with lung oedema. 2) The second model consisted of lipopolysaccaride ((LPS) Escherichia coli endotoxin 055:B5; Sigma Chemicals, L'lle d'Abeau Chêne, France) in alveolar instillation [8]. The dose instilled induced a 
similar alveolar-neutrophil influx subsequent to $P$. aeruginosa instillation, however, almost no severe injury was evident (no mortality, mild lung oedema). 3) The third model consisted of intraperitoneal injection of alpha naphthylthiourea (ANTU; Kodak, Rochester, NY, USA; $10 \mathrm{mg} \cdot \mathrm{kg}^{-1}$, dissolved in dimethylsulphoxide (DMSO)). This injury model is characterised by the presence of lung oedema contrasting with the absence of alveolar-neutrophil influx [10].

Controls groups were: 1) alveolar instillation of saline ( $P$. aeruginosa and LPS vehicle); and 2) intraperitoneal injection of DMSO (ANTU vehicle).

Rats were sacrificed at the sixth or the 24th hour. Lung injury was assessed based on: 1) cell influx into alveolar spaces (BAL cell counts); 2) pulmonary oedema (wet-to-dry weight lung (W:D) ratio); and 3) static compliance.

In additional experiments, a pretreatment of animals with the specific sPLA2-IIA inhibitor 3 acetamine-1-benzyl-2 ethylindolyl-5 oxypropane phosphonic acid (LY311727; ELI Lilly Indianapolis, IN, USA) was conducted. Rats were administered with $8 \mathrm{mg} \cdot \mathrm{kg}^{-1}$ of LY311727 inhibitor or its vehicle DMSO intraperitoneally immediately before instillation with $P$. aeruginosa. Rats were sacrificed at the sixth or 24 th hour after bacterial challenge. For the group studied at the 24th hour, animals received two doses of inhibitor; once before instillation and the second injection $12 \mathrm{~h}$ after instillation.

\section{Preparation of bacterial inoculum}

A nonmucoid $P$. aeruginosa strain (serotype 11) was used. On the day of the experiment, the bacteria were centrifuged, washed, and finally resuspended in saline at a concentration of $2-4 \times 10^{9}$ bacteria $\cdot \mathrm{mL}^{-1}$.

\section{Alveolar instillation of lipopolysaccharide or Pseudomonas aeruginosa}

Alveolar instillation of bacterial inoculum or LPS was performed as described previously [8]. Briefly, under halothane anaesthesia, the trachea was exposed to administer $1 \mathrm{mg} \cdot \mathrm{kg}^{-1}$ of LPS (volume of $0.5 \mathrm{~mL} \cdot \mathrm{kg}$ body weight ${ }^{-1}$ ) or $0.5 \mathrm{~mL} \cdot \mathrm{kg}$ weight $^{-1}$ of bacterial inoculum in sterile physiological saline.

\section{Determination of static compliance}

Mechanical ventilation was performed as reported previously [11], and pressure/volume curves for the respiratory system were plotted to determine static compliance as described by Rossi et al. [12].

\section{Arterial blood gas measurements}

Aortic puncture was performed at the time of sacrifice and arterial blood gases were measured.

\section{Bronchoalveolar lavage}

In these experiments, BAL was performed with the whole right lung, W:D lung ratio was performed with the basal part of the left lung, and the apical part of the left lung was excised, frozen in liquid nitrogen and stored at $-80^{\circ} \mathrm{C}$ until use. The left bronchus was clamped to allow BAL of the right lung (middle and caudal lobes), using a tracheotomy tube. Five 4-mL aliquots of $37^{\circ} \mathrm{C}$, sterile, pyrogen-free, $0.9 \%$ saline were flushed through the tracheotomy tube. The five fractions were recovered and pooled. The total number of cells was counted using a standard haemocytometer. The BAL fluid was then centrifuged at $300 \times g$ for $7 \mathrm{~min}$. The supernatant was collected and stored at $-80^{\circ} \mathrm{C}$.

\section{Wet-to-dry lung weight ratio}

The freshly harvested left lung was separated, weighed, then dried at $40^{\circ} \mathrm{C}$ for $72 \mathrm{~h}$, and finally weighed again. The $\mathrm{W}: \mathrm{D}$ ratio was calculated by dividing the wet weight by the dry weight.

\section{Preparation of rat lung homogenates}

Lungs were excised, frozen in liquid nitrogen and stored at $-80^{\circ} \mathrm{C}$ until use. Lung homogenates were prepared as described previously and then membrane fractions were used for the determination of sPLA2-IIA activity (see below) [4].

\section{Bacterial culture}

The concentration of bacteria was quantified as described [9], results were expressed as colony-forming units per gram of tissue or millilitre of BAL fluid.

\section{Measurement of type-IIA secretory phospholipase A2 activity}

The measurement of sPLA2-IIA activity was carried out using the fluorometric assay that has been shown to be selective for sPLA2 as described previously [13]. Briefly, the fluorescent phospholipid substrate was dried under nitrogen and suspended in ethanol at a concentration of $0.2 \mathrm{mM}$. Vesicles were prepared by mixing the ethanol solution and the fluorescent phospholipid with a buffer solution containing $50 \mathrm{mM}$ Tris- $\mathrm{HCl}, 100 \mathrm{mM} \mathrm{NaCl}, 1 \mathrm{mM}$ ethyleneglycol-bis( $\beta$-aminoethylether)- $\mathrm{N}, \mathrm{N}, \mathrm{N}^{\prime}, \mathrm{N}^{\prime}$-tetraacetic acid (EGTA) with a $\mathrm{pH}$ of 7.5. The substrate solution was then mixed with $10 \%$ fatty-acid free, bovine serum albumin and aliquots from BAL fluids and lung membranes. The reactions were initiated with $\mathrm{CaCl}_{2}$ at a $10 \mathrm{mM}$ final concentration. The fluorescence measurements were performed with a Jobin et Yvon JY3D spectrofluorometer (Stamnore, UK) equipped with a Xenon lamp. To verify whether the observed activity was due to sPLA2-IIA, samples from BAL fluids and lung homogenates were preincubated with sPLA2-IIA inhibitor LY311727 before the enzymatic assay.

\section{Extraction and analysis of type-IIA secretory phospholipase A2 messenger ribonucleic acid levels}

Total ribonucleic acid (RNA) was extracted from lung tissues as described previously [3]. The RNA (10 $\left.\mu \mathrm{g} \cdot \mathrm{lane}^{-1}\right)$ was electrophoresed on a $1 \%$ agarose gel by the formaldehyde method and then transferred onto nylon membranes (Amersham, Buckingham, UK). Full-length rat sPLA2-IIA complementary deoxyribonucleic acid (cDNA) was labelled with $\alpha-\left({ }^{32} \mathrm{P}\right)$ deoxycytidine triphosphate (ICN Biochemicals, Orsay, France) using a random primer-labelling system (Amersham). The blots were hybridised overnight at $68^{\circ} \mathrm{C}$ and autoradiography was performed. Blots were washed off and rehybridised with mouse $\beta$-actin cDNA at $65^{\circ} \mathrm{C}$ as an internal control.

\section{Surfactant phospholipid quantitation and analysis of fatty-acid release}

Lipids were extracted from BAL fluids, evaporated to dryness and their phosphorous content determined as 
described previously [4]. Large (LA) and small (SA) aggregates were separated and their ratio calculated as described previously [14]. Fatty acids were extracted according to the procedure modified by TsuJISHITA et al. [14] and methylated with diazomethane. The methylated derivatives were separated by gas chromatography on a capillary column containing Supelcowax 10 bonded phase (diameter $0.32 \mathrm{~mm}, 30 \mathrm{~m}$ long (Supelco)) on a Hewlett Packard 5890 Series II gas chromatograph (Hewlett Packard, Issy les Moulineaux, France) and detected by mass spectrometry (Nermag 10-10C).

\section{Statistics}

Results are expressed as means \pm SEM. Intergroup comparisons were performed using nonparametric methods, namely Kruskal-Wallis rank-sign analysis of variance, the MannWhitney test, or Spearman's rank correlation coefficient as appropriate. Statistical significance was defined as $\mathrm{p}<0.05$.

\section{Results}

\section{Rat acute lung injury models}

Indexes of lung injury. Comparison of the three models of ALI (table 1) showed that the different indexes of lung injury progressed in parallel since at least some degree of correlation between these indexes was evident (table 2).

Type-IIA secretory phospholipase A2 activity and surfactant phospholipid quantitation. Alveolar instillation of LPS or live bacteria led to a similar level of neutrophil influx into alveolar spaces and decreased static compliance. During both injuries, sPLA2-IIA messenger ribonucleic acid (mRNA) was upregulated in lung homogenate (fig. 1), which resulted in a parallel increase in sPLA2-IIA activity (table 1). This was accompanied by a marked loss in surfactant phospholipid content $24 \mathrm{~h}$ after LPS or bacterial instillation (fig. 2). No significant change in this content was observed $6 \mathrm{~h}$ after instillation (data not shown). In LPS-, bacteria-instilled rats and healthy rats, a negative correlation between the increased concentration of sPLA2-IIA activity in BAL fluid and the decrease in compliance was evident (fig. 3). By contrast, ANTU-induced injury did not lead to alveolar-neutrophil recruitment or to an increase in sPLA2-IIA mRNA expression and activity (table 1 and fig. 1); it also did not lead to surfactant-phospholipid degradation (data not shown). When examining all groups of rats, both sPLA2-IIA activity and BAL cell counts were positively correlated (fig. 4a). Furthermore, static compliance was negatively correlated with sPLA2IIA activity in lung homogenate (fig. 4b).

Gas chromatography/mass spectrometry analysis of the BAL fluid from bacteria-treated rats showed an increase in the levels of free fatty acids, with palmitic and linolenic acids being the major fatty acids released (fig. 2). The levels of palmitic acid were closely correlated with the increase in

Table 2. - Correlations between the indexes of lung injury

\begin{tabular}{llrr}
\hline Index & \multicolumn{1}{c}{ Index } & rho value & p-value \\
\hline${\mathrm{Pa}, \mathrm{O}_{2}}$ & W:D lung ratio & -0.28 & 0.15 \\
${\mathrm{~Pa}, \mathrm{O}_{2}}_{\mathrm{Compliance} \text { rs }}$ & Compliance rs & 0.23 & 0.19 \\
W:D lung ratio & BAL fluid cell count & -0.41 & 0.02 \\
Compliance rs & BAL fluid cell count & -0.47 & 0.04 \\
\hline
\end{tabular}

$\mathrm{Pa}_{\mathrm{a}} \mathrm{O}_{2}$ : arterial oxygen tension; W:D: wet-to-dry weight lung ratio; rs: Spearman rank correlation coefficient; BAL: bronchoalveolar lavage. 

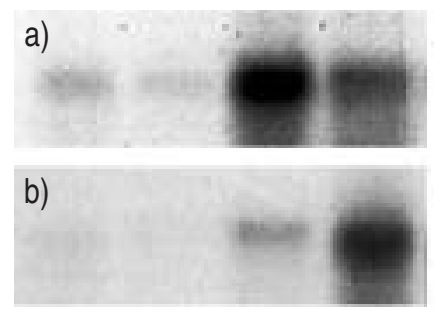

SPLA2-IIA

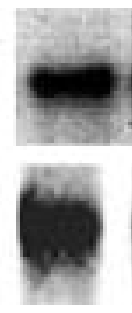

Control

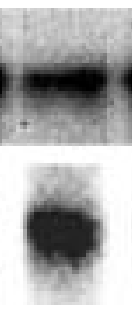

ANTU

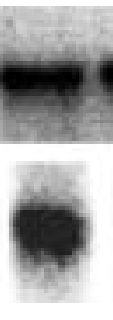

LPS

$\beta$-actin

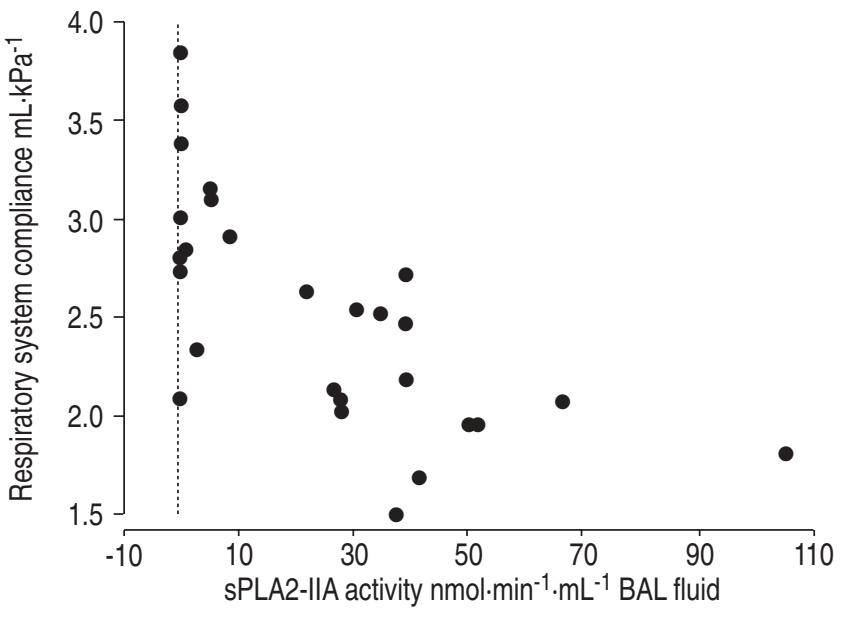

Fig. 3. - Correlation between static respiratory system compliance and type-II secretory phospholipase A2 (sPLA2-IIA) activity in bronchoalveolar lavage (BAL) fluids of instilled rats (lipopolysaccharide or bacteria or saline). A negative correlation between these two parameters was observed: $r h o=0.72, p<0.001$. Dotted line represents zero.
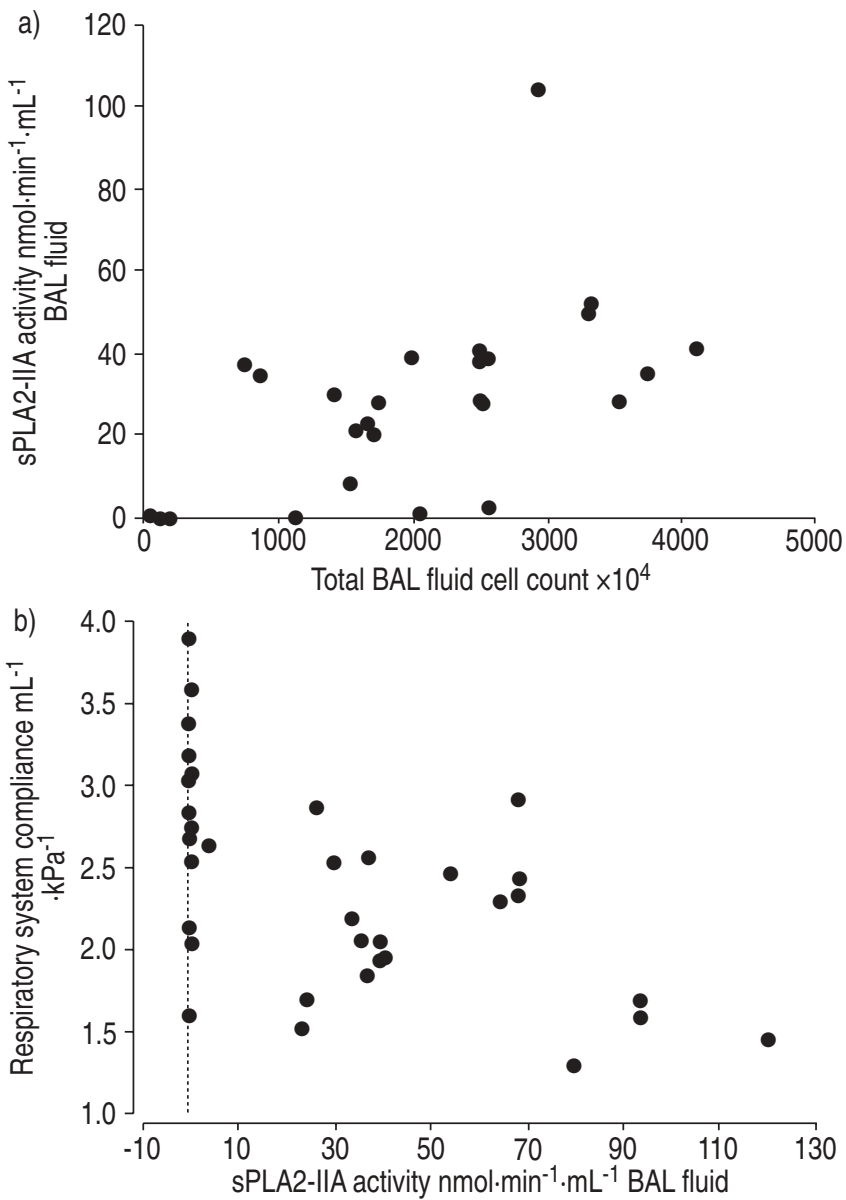

Fig. 4.-Correlation between a) type-II secretory phospholipase A2 (sPLA2-IIA) activity and total cell count in bronchoalveolar lavage (BAL) fluids (rho=0.74, $\mathrm{p}<0.0001$ ), and b) homogenate sPLA2-IIA activity and static respiratory system compliance of injured rats (alpha naphthylthiourea, lipopolysaccharide or bacteria) and control rats ( $\mathrm{rho}=-0.37, \mathrm{p}<0.05)$. Dotted line represents zero.

of any physiological measure (even bacterial culture) in bacterial-instilled rats was apparent, irrespective of whether or not they received the inhibitor (table 1). 

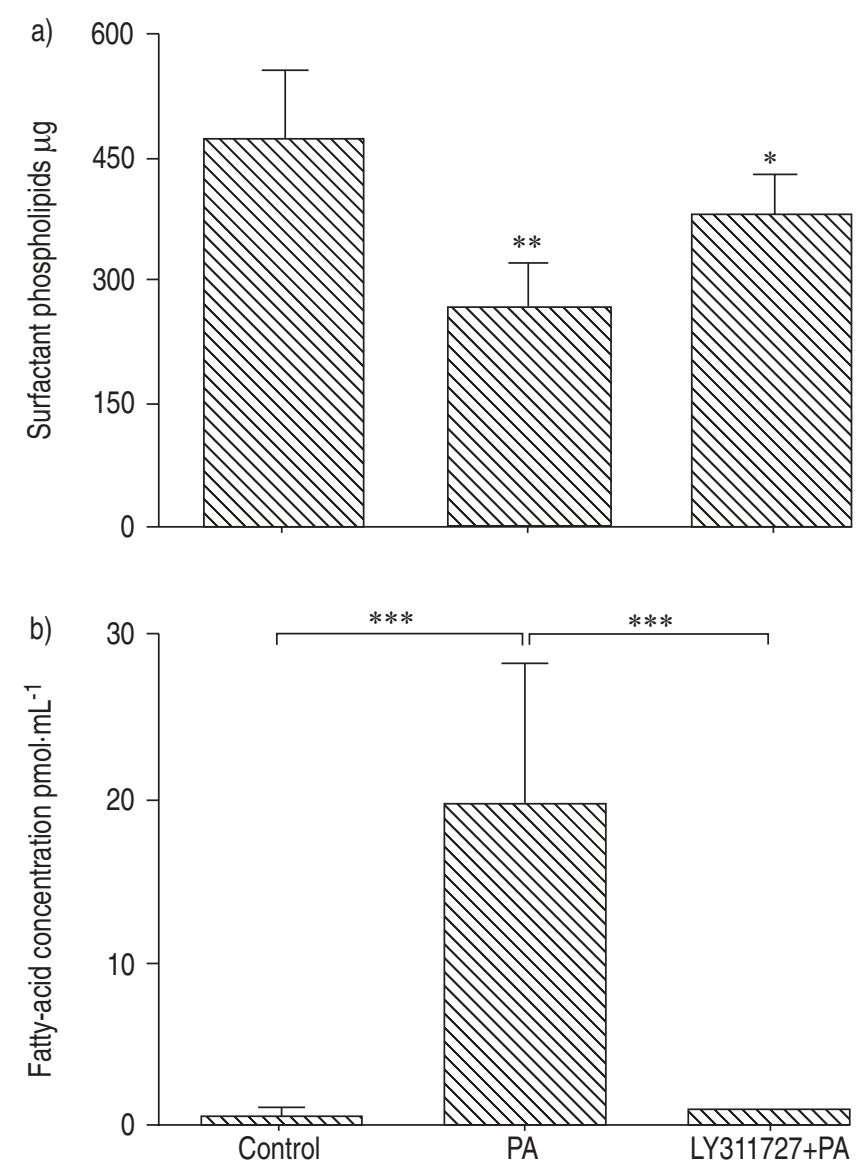

Fig. 5.-Biochemical effect of a specific type-II secretory phospholipase A2 (sPLA2-IIA) inhibitor administration during Pseudomonas aeruginosa (PA)-induced lung injury. Animals were treated intraperitoneally with $8 \mathrm{mg} \cdot \mathrm{kg}^{-1}$ of specific sPLA2-IIA inhibitor LY311727 or its vehicle dimethylsulphoxide, immediately before and $12 \mathrm{~h}$ after PA instillation. Bronchoalveolar lavage (BAL) was performed $24 \mathrm{~h}$ later, and then a) surfactant phospholipid content and b) free fatty acid levels were analysed in cell-free BAL. *: $p<0.05$ compared with both control and PA rats; ${ }^{\#}: \mathrm{p}<0.05$ compared with control animals; $* * *: \mathrm{p}<0.001$.

The authors next investigated whether hydrolysis of surfactant phospholipids in $P$. aeruginosa instilled rats is accompanied by a change in the ratio of LA to SA of surfactant. LA represented $103 \%(94-112, n=2)$ and $33 \pm 6 \%(n=4)$ of total aggregates in saline and $P$. aeruginosa instilled animals, respectively $(\mathrm{p}<0.05)$. When animals were pretreated with LY311727 before instillation of $P$. aeruginosa the percentage of LA was $38 \pm 13 \quad(n=3$, nonsignificantly different from percentage of $P$. aeruginosa instilled rats without inhibitor).

\section{Discussion}

Surfactant deficiency has been established as the primary cause of the respiratory failure in infant respiratory distress syndrome, and transbronchial application of surfactant preparations has become the gold standard for the treatment of this disorder. In ARDS, however, surfactant deficiency seems not to be of major importance; rather, a broad spectrum of biochemical and biophysical surfactant abnormalities contributes to respiratory failure $[1,2]$. These included alteration of the phospholipid and fatty-acid profile, decreased levels of surfactant apoproteins, reduced content of large surfactant aggregates, inhibition of surfactant function by leaked plasma proteins and inhibition by inflammatory mediators. Among these factors, elevated levels of sPLA2IIA could participate in inhibition of surfactant function $[5,6]$. The authors have previously shown that sPLA2-IIA is mainly synthesised by alveolar macrophage under tumour necrosis factor (TNF)- $\alpha$ control [3], and participates in surfactant degradation during endotoxin-induced ALI in guinea-pigs [4]. However, the functional consequences of the increased sPLA2-IIA activity were not assessed. Consequently, three models of rat ALI were investigated to assess whether increased sPLA2-IIA activity is a characteristic of ALI, and whether this activity correlates with the parameters of injury.

The three rat models of ALI were characterised by various degree of lung injury as shown by a significant decrease in respiratory static compliance and a significant increase in lung oedema compared with healthy rats. Interestingly, static compliance was correlated with both lung oedema and alveolar cell counts (table 2). Accordingly with this latter finding, INGENITO et al. [15] have demonstrated a correlation between compliance (ex-vivo experiments on lung strip from acid-instilled animals) and cellular infiltration. Furthermore, activated leukocytes have been shown to cause surfactant dysfunction in vitro by serine protease-mediated degradation of surfactant protein-A [16].

\section{Increased type-IIA secretory phospholipase A2 activity is linked to alveolar inflammation}

The main difference between the three models of ALI was that alveolar-neutrophil influx was observed only after LPS- or bacterial instillation, as demonstrated previously $[8,10]$. The authors have previously shown that an inflammatory response occurs in both LPS- and bacteria-instilled lungs, at least in term of TNF- $\alpha$ secretion [7, 17], and that its local secretion participates to alveolar liquid clearance [7, 18]. Therefore, it could explain the absence of significant persistent oedema in these models at the 24th hour. By contrast, no neutrophil influx was apparent during ANTUinduced injury. This difference could be explained by the lack of alveolar macrophage activation during ANTU-induced injury, probably resulting in the absence of neutrophilchemokine secretion. Along this line, it has recently been demonstrated that $\mathrm{TNF}-\alpha$ secretion by alveolar macrophages is even downregulated after ANTU injury [10]. Furthermore, the relationship between alveolar sPLA2-IIA activity levels and alveolar cell counts further reinforce the link between activated macrophages and/or recruited neutrophils [19] and this enzyme secretion.

\section{Functional consequence of increased type-IIA secretory phospholipase A2 activity}

The contribution of SPLA2-IIA activity to the functional decrease in static compliance is suggested by the correlation between the increase in enzyme activity and the decrease in compliance in LPS- and bacteria-instilled rats and the evidence for surfactant degradation. This latter finding, together with surfactant-function alteration, has previously been shown in the same animal model by VANDERZWAN et al. [20]. The present results extend their results, inasmuch as surfactant phospholipids are shown to be a major target of sPLA2-IIA. Accordingly, high concentrations of palmitic acid, the major fatty acid esterified in surfactant phospholipids, was found in BAL fluids of bacteria-instilled rats. An increase in the concentrations of other fatty acids (linoleic and linolenic acids) was also observed which suggests that sPLA2IIA hydrolysed not only surfactant phospholipids, but also 
cell membrane phospholipids. Administration of LY311727, a specific inhibitor of sPLA2-IIA activity, markedly reduced the hydrolysis of surfactant phospholids without modifying bacterial clearance (data not shown) in this ALI model. Nevertheless, other factors are probably involved in lung injury since LY311727 was unable to restore a normal static compliance. Along this line, static compliance was correlated with alveolar cell counts, therefore other mediators secreted by these activated cells could have participated to the alterations of lung mechanics. In contrast to the present authors' findings, FURUE et al. [21] recently demonstrated the beneficial effects of an sPLA2-IIA inhibitor (S-5920/ LY315920Na) on lung mechanics in an animal model of ALI induced by oleic acid. The main difference from the present study's experimental design was that FURUE et al. [21] assessed injury parameters during the first $6 \mathrm{~h}$ following the onset of oleic acid administration.

The results also showed that bacterial instillation markedly decreased the ratio of LA to SA. However, administration of sPLA2-IIA inhibitor LY311727 failed to prevent this decrease, which suggests that hydrolysis of surfactant phospholipids by SPLA2-IIA may not play a major role in the change of ratio aggregates observed in $P$. aeruginosatreated rats. A limitation of this study was the absence of direct measurement of functional consequence of surfactant hydrolysis. However, both in-vitro and in-vivo studies have demonstrated that this hydrolysis significantly increased surface tension of surfactant $[22,23]$.

In summary, rat models of ALI demonstrated that an increase in both sPLA2-IIA activity and surfactant degradation occurred when alveolar inflammation was present. This was associated with a decrease in static compliance. This study demonstrates the induction of sPLA2-IIA and its involvement in surfactant degradation in an animal model of ARDS induced by bacterial infection. This model is of more pathophysiological relevance than the previously reported model using LPS as the inducer of ALI.

It would be of interest to investigate whether lipopolysaccaride is the first bacterial component involved in the induction of type-II secretory phospholipase A2. Animals treated with a specific inhibitor of type-II secretory phospholipase A2 activity demonstrated no improvement in physiological parameters despite a biochemical effect, suggesting that its activity (and probably surfactant degradation) is only one of the multiple factors involved in acute lung injury pathophysiology. Consequently, further experiments are warranted to explore whether type-II secretory phospholipase A2 activity inhibition could be associated to other treatments as exogenous surfactant.

Acknowledgements. The authors would like to thank H. Arita (Shionogi Research laboratory, Osaka, Japan) for the generous gift of the rat sPLA2-IIA cDNA.

\section{References}

1. Lewis JF, Jobe AH. Surfactant and the adult respiratory distress syndrome. Am Rev Respir Dis 1993; 147: 218-233.

2. Gunther A, Ruppert C, Schmidt R, et al. Surfactant alteration and replacement in acute respiratory distress syndrome. Respir Res 2001; 2: 353-364.

3. Arbibe L, Vial D, Rosinski-Chupin I, et al. Endotoxin induces expression of type II phospholipase A2 in macrophages during acute lung injury in guinea pigs: involvement of TNF-alpha in lipopolysaccharide-induced type II phospholipase A2 synthesis. J Immunol 1997; 159: 391-400.

4. Arbibe L, Koumanov K, Vial D, et al. Generation of lysophospholipids from surfactant in acute lung injury is mediated by type-II phospholipase A2 and inhibited by a direct surfactant protein A-phospholipase A2 protein interaction. J Clin Invest 1998; 102: 1152-1160.

5. Touqui L, Arbibe L. A role for phospholipase A2 in ARDS pathogenesis. Mol Med Today 1999; 5: 244-249.

6. Touqui L, Alaoui-El-Azher M. Mammalian secreted phospholipases A2 and their pathophysiological significance in inflammatory diseases. Curr Mol Med 2001; 1: 739-754.

7. Rezaiguia S, Garat C, Delclaux C, et al. Acute bacterial pneumonia in rats increases alveolar epithelial fluid clearance by a tumor necrosis factor-alpha-dependent mechanism. J Clin Invest 1997; 99: 325-335.

8. Delclaux C, Rezaiguia-Delclaux S, Delacourt C, Brun-Buisson C, Lafuma C, Harf A. Alveolar neutrophils in endotoxininduced and bacteria-induced acute lung injury in rats. $\mathrm{Am} \mathrm{J}$ Physiol 1997; 273: L104-L112.

9. Jean D, Rezaiguia-Delclaux S, Delacourt C, et al. Protective effect of endotoxin instillation on subsequent bacteriainduced acute lung injury in rats. Am J Respir Crit Care Med 1998; 158: 1702-1708.

10. Azoulay E, Attalah H, Yang K, et al. Neutrophils mediate the exacerbation by G-CSF of prior acute lung injury. Crit Care Med 2002; 30: 2115-2122.

11. Lorino AM, Benichou M, Kochi T, Lorino H, Milic-Emili J, Harf A. Comparison of the constant flow and occlusion methods for assessment of bronchoconstriction in guineapigs. Eur Respir J 1989; 2: 84-89.

12. Rossi A, Gottfried SB, Higgs BD, Zocchi L, Grassino A, Milic-Emili J. Respiratory mechanics in mechanically ventilated patients with respiratory failure. J Appl Physiol 1985; 58: 1849-1858.

13. Hidi R, Vargaftig BB, Touqui L. Increased synthesis and secretion of a 14-kDa phospholipase A2 by guinea pig alveolar macrophages. Dissociation from arachidonic acid liberation and modulation by dexamethasone. J Immunol 1993; 151: 5613-5623.

14. Tsujishita Y, Asaoka Y, Nishizuka Y. Regulation of phospholipase A2 in human leukemia cell lines: its implication for intracellular signaling. Proc Natl Acad Sci USA 1994; 91: 6274-6278.

15. Ingenito EP, Mark L, Davison B. Effects of acute lung injury on dynamic tissue properties. J Appl Physiol 1994; 77: 2689-2697.

16. Schochett $\mathrm{P}$, Mora $\mathrm{R}$, Mark L, Butler M, Ingenito EP. Calcium-dependent degradation of surfactant protein A by activated neutrophils due to serine proteases. Exp Lung Res 1999; 25: 595-616.

17. Van de Louw A, Jean D, Frisdal E, et al. Neutrophil proteinases in hydrochloric acid- and endotoxin-induced acute lung injury: evaluation of interstitial protease activity by in situ zymography. Lab Invest 2002; 82: 133-145.

18. Garat C, Rezaiguia S, Meignan M, et al. Alveolar endotoxin increases alveolar liquid clearance in rats. $J$ Appl Physiol 1995; 79: 2021-2028.

19. Weiss J, Inada M, Elsbach P, Crowl RM. Structural determinants of the action against Escherichia coli of a human inflammatory fluid phospholipase A2 in concert with polymorphonuclear leukocytes. J Biol Chem 1994; 269: 26331-26337.

20. Vanderzwan J, McCaig L, Mehta S, et al. Characterizing alterations in the pulmonary surfactant system in a rat model of Pseudomonas aeruginosa pneumonia. Eur Respir J 1998; 12: $1388-1396$.

21. Furue S, Mikawa K, Nishina K, et al. Therapeutic timewindow of a group IIA phospholipase A2 inhibitor in rabbit acute lung injury: correlation with lung surfactant protection. Crit Care Med 2001; 29: 719-727.

22. Holm BA, Keicher L, Liu MY, Sokolowski J, Enhorning G. Inhibition of pulmonary surfactant function by phospholipases. J Appl Physiol 1991; 71: 317-321.

23. Schrama AJ, de Beaufort AJ, Sukul YR, Jansen SM, Poorthuis BJ, Berger HM. Phospholipase A2 is present in meconium and inhibits the activity of pulmonary surfactant: an in vitro study. Acta Paediatr 2001; 90: 412-426. 